\title{
Towards Fabless Silicon Photonics
}

\author{
Pieter Dumon \\ Ghent University - IMEC, Department of Information Technology, \\ Sint-Pietersnieuwstraat 41,9000 Gent, Belgium \\ Pieter.Dumon@imec.be
}

\begin{abstract}
A well-defined technology platform is necessary for a broader take-up of silicon photonics. We are working towards such platform, with a generic integration technology, a well-defined design interface, and integration with the full food chain.
\end{abstract}

To allow submicron silicon photonics research to move to the IC and system design level, to support a wider range of users as well as a wide range of applications, a generic integration technology platform is necessary. With the ePIXfab, the European silicon photonics platform, we are working towards such a platform, based on high-end CMOS facilities and fabs. The most compelling argument in favor of silicon photonics is that one can re-use the CMOS fabs, which run stable, high-yield processes for volume fabrication. Such expensive fabs can only be maintained by large integrated manufacturers or by professional foundries, however. Therefore, fabless access to silicon photonics is essential to allow for a dynamic environment. A key concept that is necessary to drive a wider adoption is the separation between technology and design, which is essential in electronic integrated circuits. Limiting the technology options allows for running such well-controlled processes, and allows for a large freedom in the design direction. A well-specified design interface is necessary, however. In addition, design for manufacturing and technology-aware design are already as important for photonics as it is becoming now for electronics.

Today, several building blocks in the silicon photonics food-chain are missing or are only partly developed. IMEC (B) and CEA-LETI (F) started ePIXfab to address these issues. Both institutes have demonstrated basic silicon photonic building blocks, such as passive components, sources, modulators and detectors. ePIXfab now organizes access to this technology through cost sharing fabrication runs for research and prototyping. The technology portfolio contains passives as well as $\mathrm{SiGe}$ processes for active devices. We are further developing this platform, targeting an expanded technology portfolio, management of the complexity, and reducing the effort and risk for the user through improvement of the design interface and education. In addition, it essential for the users to have access the full food-chain in order to enable system-level research and development. Therefore, ePIXfab is cooperating with other service providers and researchers for development of design libraries, packaging approaches, and postprocessing.

\section{Acknowledgement}

This work is supported by the European Community through the FP7 PhotonFAB (contract 224232) and HELIOS (contract 224312) projects. 

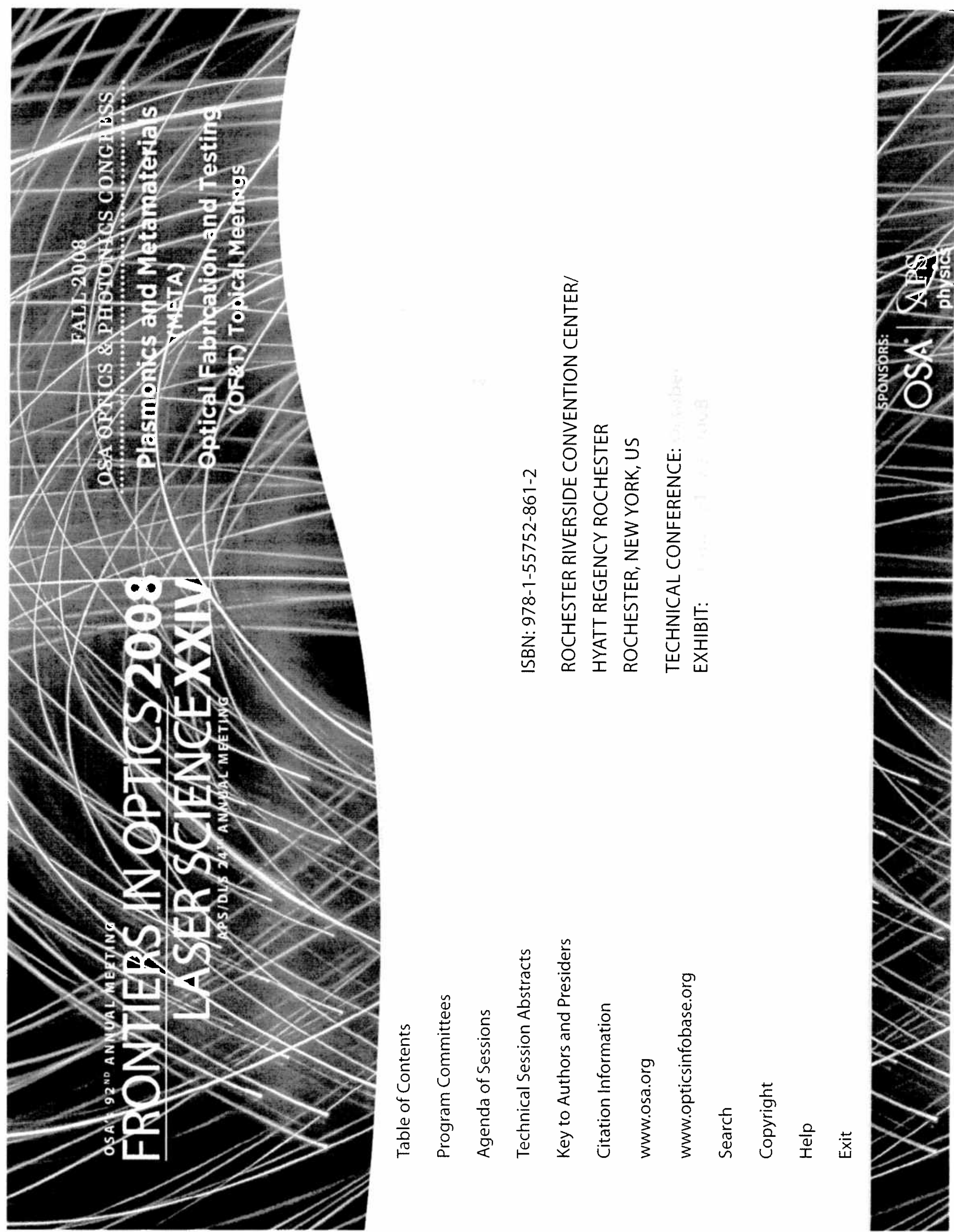